\title{
Le myoépithéliome : une tumeur rare des glandes salivaires. A propos d'un cas.
}

\author{
Myoepithelioma of minor salivary glands. A case report
}

SOPHIE BAHI*, ANDRÉ WARTER**, AHMED FEKI*

\section{RÉSUMÉ}

Le myoépithéliome est une tumeur bénigne rare des glandes salivaires aujourd'hui considérée comme une entité nosologique propre. Cet article rapporte le cas d'un myoépithéliome des glandes salivaires accessoires découvert fortuitement chez un homme de 36 ans. L'aspect clinique de la lésion (contours bien définis, recouverte d'une muqueuse saine) suggère le diagnostic de tumeur bénigne des glandes salivaires. Les examens microscopiques et immunohistochimiques ont confirmé la nature myoépithéliale de la tumeur. Le diagnostic de ce néoplasme reste toutefois controversé en raison de l'éventail des caractéristiques cliniques, histologiques et cellulaires subtiles qu'il peut revêtir. La connaissance de ces modèles architecturaux et des profils immunohistochimiques spécifiques présentés dans ce travail est également cruciale pour identifier précisément ces tumeurs et éviter un diagnostic erroné. (Med Buccale Chir Buccale 2003; 9: 113-118)

médecine buccale chirurgie buccale VOL. $9, \mathrm{~N}^{\circ}$ 2003 page 113

mots clés : Myoépithéliome, tumeur des glandes salivaires.

\section{SUMMARY}

Myoepithelioma, presently classified as a « distinct » clinical and pathological entity, is a rare benign salivary gland neoplasm. A case, which occurred in the minor salivary glands in a 36 years-old man, is reported. Following clinical inspection showing a normally limited swelling without color change of the muccosa, a benign salivary gland neoplasm was suspected. Histological examinations as well as immunochemical investigations confirmed the myoepithelial nature of the tumor. However, the diagnosis of this neoplasm remains controversial since a wide range of clinical and histological features can be display. Cytoarchitectural patterns and immunochemical profiles are also crucial for identification and avoid potential misdiagnosis. (Med Buccale Chir Buccale 2003; 9: 113-118)

key words : Myoepithelioma, salivary gland neoplasm.

\footnotetext{
* Département de Médecine buccale et de Chirurgie buccale - Hôpitaux Universitaires de Strasbourg-F

** Institut d'Anatomie Pathologique, Faculté de Médecine de Strasbourg-F
}

Demande de tirés à part :

Sophie Bahi Département MBCB Faculté de Chirurgie Dentaire 1, Place de l'Hôpital 67091 Strasbourg

Article reçu le 21 janvier 2003. Accepté pour publication le 2 avril 2003. 
médecine buccale chirurgie buccale
Le myoépithéliome est une tumeur bénigne des glandes salivaires aujourd'hui considérée comme une entité nosologique propre. Bien qu'assez rare, (moins de $1 \%$ des tumeurs des glandes salivaires) ${ }^{[1]}$ cette tumeur identifiée par Sheldon en 1941 doit être connue de l'odontologiste, du chirurgien mais aussi du pathologiste puisque l'attitude thérapeutique (exérèse complète de la tumeur suivie d'une surveillance régulière) est dictée par ce diagnostic. II s'agit d'un diagnostic difficile et parmi la surabondance des variétés et sous variétés des tumeurs de glandes salivaires, la lésion peut être confondue tant avec d'autres lésions bénignes susceptibles de récidiver qu'avec des tumeurs malignes.

Cet article a pour objectif de décrire les aspects cliniques et histologiques des myoépithéliomes au travers d'un cas développé à partir des glandes salivaires accessoires palatines.

\section{OBSERVATION}

Le patient, 36 ans se présente en décembre 2000 au Département de Médecine Buccale et de Chirurgie Buccale pour remise en état de la cavité buccale et réhabilitation prothétique. Ce patient ne présente aucun antécédent médicochirurgical majeur et ne suit aucun traitement médicamenteux. L'examen clinique exobuccal est normal. L'examen endobuccal met en évidence (Fig. 1) :

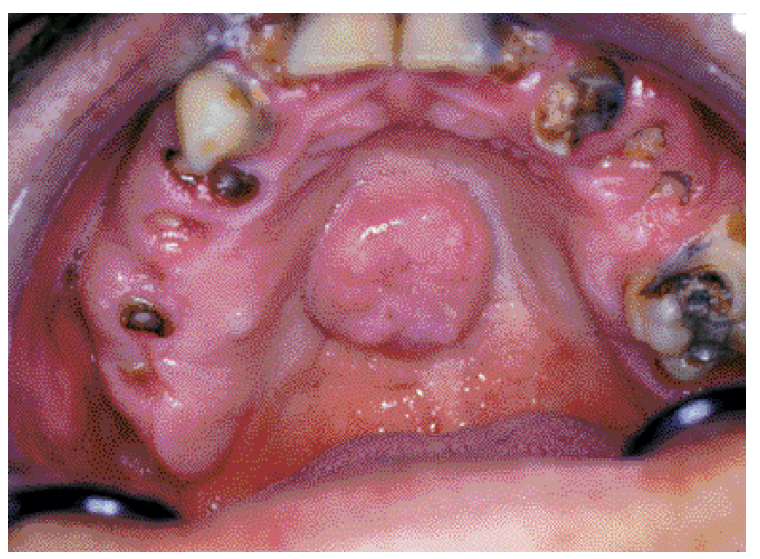

Figure 1 : Vue endobuccale : noter l'état bucco-dentaire négligé et la lésion palatine découverte fortuitement

Clinical examination revealed multiple tooth caries and totally asymptomatic palatal swelling
- un état dentaire négligé avec de nombreuses lésions carieuses et racines résiduelles ;

- une très mauvaise hygiène bucco-dentaire ;

- des dépôts tartriques importants au niveau de la région antérieure mandibulaire (indemne de lésions carieuses);

- une tuméfaction palatine.

Le cliché panoramique confirme les données de l'examen endobuccal : de nombreux foyers apicaux sur $22,25,25$ et 47 associés à une alvéolyse horizontale de la région antérieure mandibulaire. Aucun signe d'atteinte osseuse associé à la tuméfaction n'a été mis en évidence sur l'orthopantomogramme (Fig. 2).

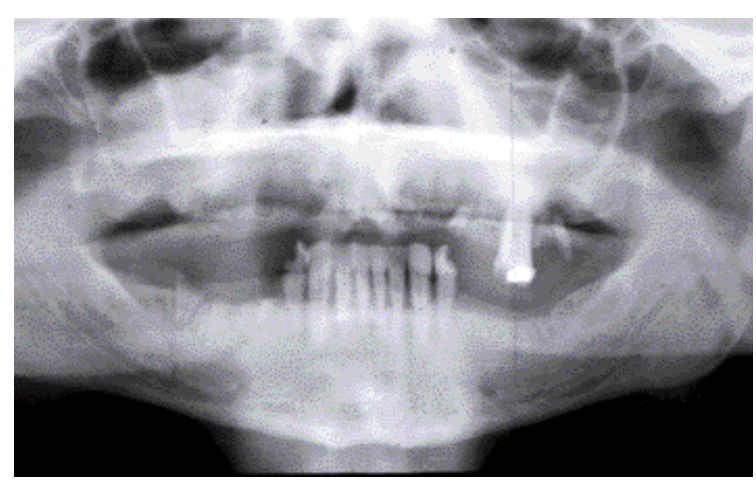

Figure 2 : Orthopantomogramme : nombreux foyers apicaux et racines résiduelles

Radiographic finding: multiple periapical inflammatory lesions

La tuméfaction palatine découverte fortuitement à l'examen clinique est en situation médiane (Fig.3).

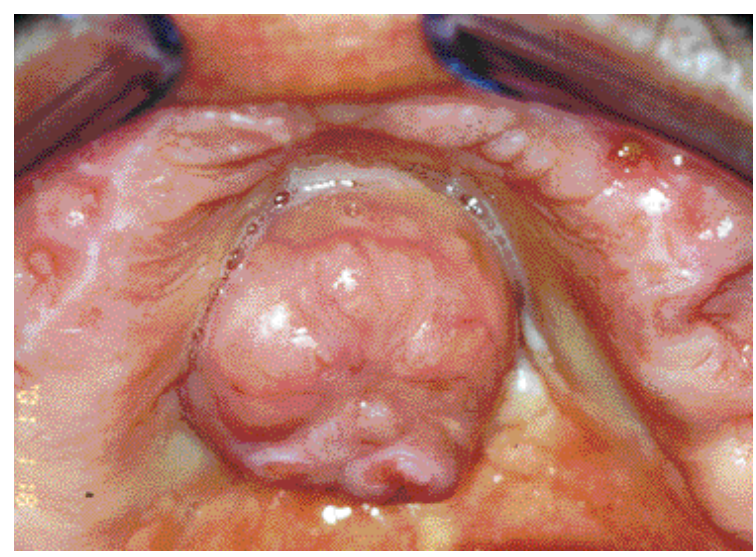

Figure 3 : Vue préopératoire : tuméfaction palatine d'implantation sessile recouverte d'une muqueuse saine

Clinical examination: well-defined palatal swelling. The overlying mucosa was normal 
De forme sphérique $(2 \times 2 \times 1,5 \mathrm{~cm})$, elle est recouverte d'une muqueuse saine et non ulcérée. Les contours sont bien définis. D'implantation sessile, la lésion présente un aspect légèrement lobulé et sa consistance est ferme sans être dure. Elle est indolore à la palpation. Elle évoluerait insidieusement depuis plus d'un an sans alerter le patient qui ne décrit aucun antécédent douloureux associé à cette lésion. L'aspect clinique de la tuméfaction suggère le diagnostic de tumeur bénigne des glandes salivaires accessoires.

Devant ce tableau clinique et pour répondre à la demande du patient, une édentation totale maxillaire et partielle mandibulaire (conservation des dents antérieures mandibulaires) est initialement envisagée. Au préalable, une empreinte d'étude maxillaire est réalisée afin de préparer une gouttière de protection thermoformée. L'exérèse de la lésion palatine est effectuée 6 semaines après les avulsions. Une anesthésie locale (Articaïne adrénalinée à 1/200000) par infiltration péri-lésionnelle suivie d'une incision circulaire (à l'aide d'un bistouri à lame froide $n^{\circ} 15$ ) a permis l'ablation de la totalité de la lésion préservant ainsi son intégrité (Fig. 4). L'hémostase locale est assurée par électrocoagulation. Elle est complétée par l'application d'oxycellulose résorbable (Surgicel ${ }^{\circledR}$ ) au niveau du site hémorragique. La prescription post-opératoire d'antalgiques (paracétamol, $3 \mathrm{~g} / \mathrm{j}$ à la demande) a été associée aux conseils alimentaires et d'hygiène bucco-dentaire habituels. L'examen anatomopathologique met en évidence un nodule de $2 \mathrm{~cm}$ de grand axe, non ulcéré, de

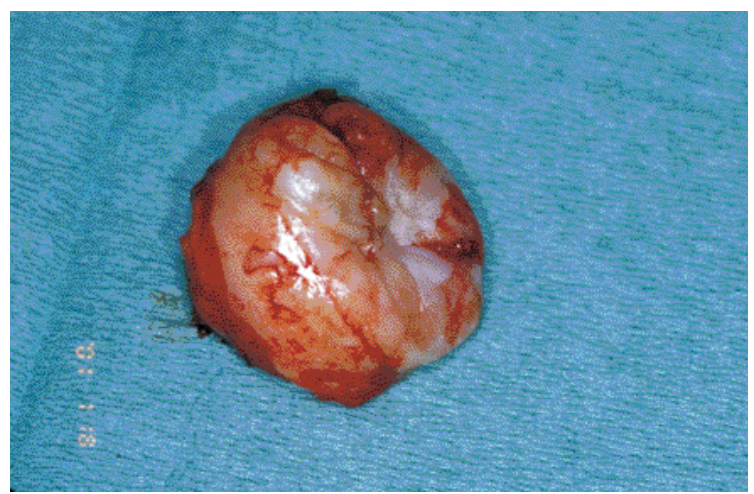

Figure 4 : Pièce opératoire : masse sphérique de consistance ferme

Macroscopical examination: the tumour featured a moderately firm consistency couleur beige homogène à la section et d'apparence bien limitée. Histologiquement, la lésion est formée de massifs, de travées et de cordons cellulaires séparés par un stroma fibro-hyalin. II n'existe en revanche aucun territoire chondroïde ou myxoïde. Les cellules tumorales sont dans leur presque totalité d'apparence plasmocytoïde (Fig.5), avec un noyau régulier déjeté par un cytoplasme abondant, éosinophile, homogène et cireux. D'autres cellules, beaucoup moins abondantes, ont un noyau comparable aux précédentes mais un cytoplasme très réduit. Enfin, quelques structures tubuleuses faites de petites cellules épithéliales s'observent très rarement au sein des massifs plasmocytoïdes. La région la plus proliférative de la lésion montre un index mitotique inférieur à une mitose pour 10 champs à fort grossissement, et il n'y a ni atypies, ni nécrose. La tumeur comporte dans sa partie superficielle et sous muqueuse une fine capsule scléreuse, et ne présente dans ces territoires aucun envahissement du tissu glandulaire salivaire voisin. II n'existe pas d'envahissement périnerveux. En revanche latéralement et en profondeur, alternent des territoires où réapparaît cette capsule et d'autres où la section chirurgicale, identifiée par teinture de la pièce, passe au contact du tissu tumoral.

L'étude immunohistochimique montre l'association d'un marquage net dans une majorité de cellules de la pankératine KL1 (Fig. 6A), de la vimentine, de la PS-100 et de l'actine muscle lisse (Fig.6B).

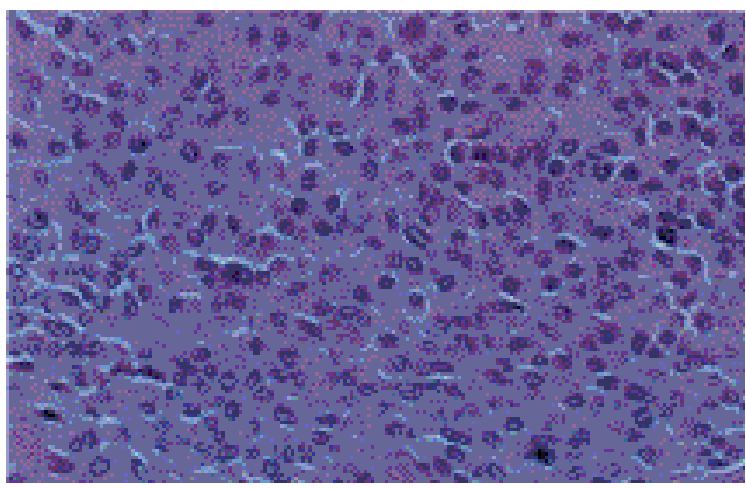

Figure 5 : Examen anatomo-pathologique : prolifération tumorale formée de cellules épithélioides et plasmocytoïdes

Histopathologic findings: tumour made of epitheliod and plasmocytoid cells proliferation médecine buccale chirurgie buccale VOL. $9, \mathrm{~N}^{\circ}$ 2003 page 115 

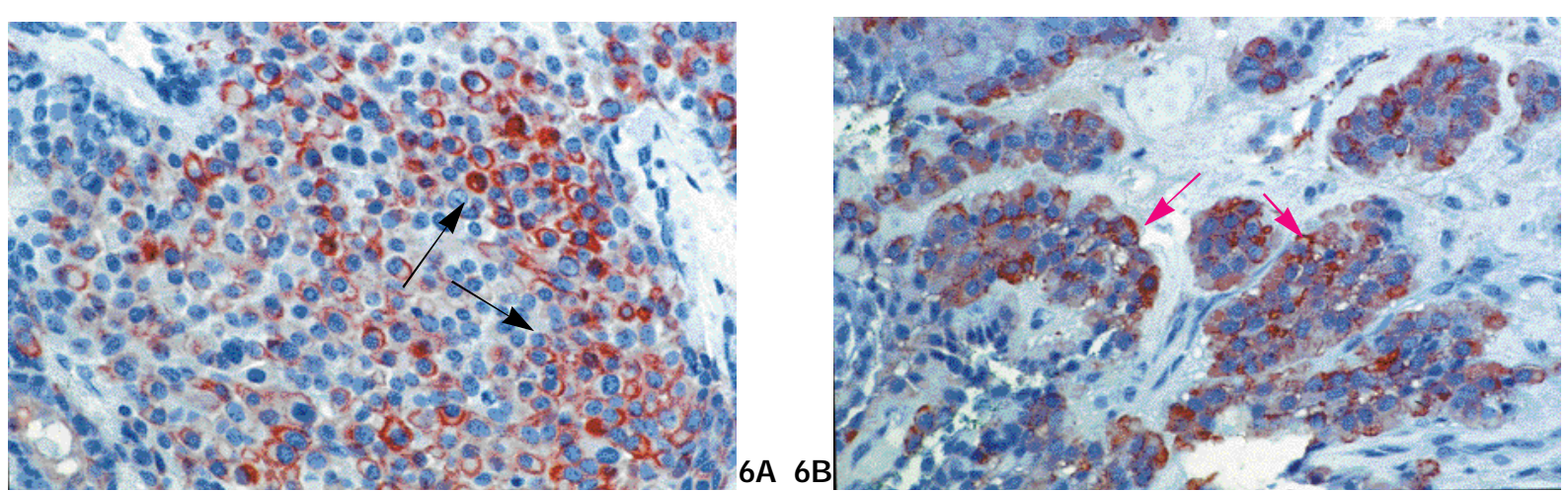

Figures 6 : Les examens immuno-histochimiques confirment le phénotype myoépithélial de la prolifération :

Figure $6 \mathrm{~A}$ : anti-kératine $(\mathrm{KL} 1)+(\rightarrow)$

Figure $6 \mathrm{~B}$ : actine musculaire $+(\rightarrow)$

Immunohistochemical investigation showed that a large part of the cells were positive with anti-keratin $(K L 1)(\rightarrow)$ and smooth muscle actin $(\rightarrow)$.

These data confirmed the myoepithelial nature of the tumour.

Les suites opératoires sont satisfaisantes. Le contrôle post-opératoire de la lésion (1 semaine après l'intervention) montre un début de réépithélialisation de la lésion à partir des berges de la plaie. Un contrôle régulier (trimestriel) du site opératoire est instauré. Un an après l'intervention aucun signe de récidive n'a été mis en évidence cliniquement (Fig. 7).

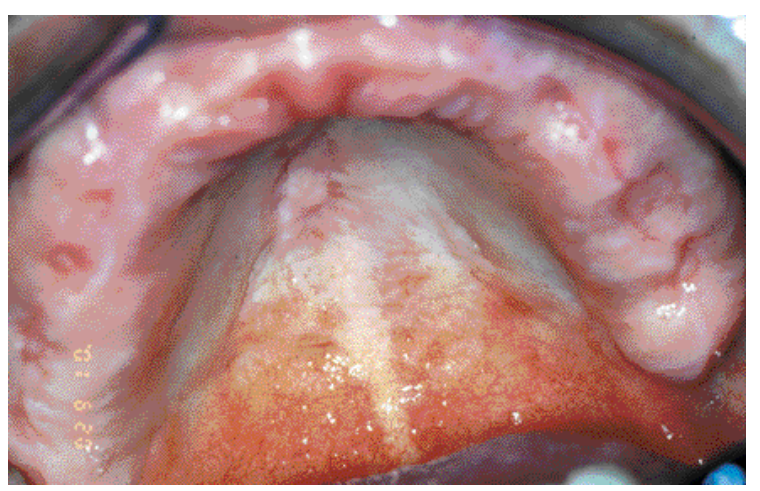

Figure 7 : Contrôle 1 an après l'intervention Clinical examination: 1 year after resection

\section{COMMENTAIRES}

Les caractères histologiques et immunohistochimiques de la lésion permettent d'identifier un myoépithéliome (ou myoépithélioma ou tumeur à cellules myoépithéliales), tumeur dont le pronostic est celui d'une tumeur mixte [1-3].

Cette tumeur est rare : elle représente moins de $1,5 \%$ des tumeurs des glandes salivaires [3], un peu plus lorsque la présence de quelques structures épithéliales est admise dans la tumeur [2]. Elle atteint indifféremment hommes et femmes. L'âge moyen de survenue, variable selon les auteurs, oscille entre 39 et 53 ans $[3,4]$. Le siège de prédilection est, par ordre décroissant, la parotide $(40 \%)$, les glandes salivaires accessoires en premier lieu palatines $(21 \%)$, et la sous mandibulaire [3,5]. II faut également signaler que, comme c'est souvent le cas en pathologie salivaire, des cas de myoépithéliome comme d'ailleurs de carcinome myoépithélial ont été découverts en différents sièges, y compris le sein [6], les tissus mous [7], la peau [8], et, ce qui n'est évidemment pas étonnant au niveau du larynx [9]. Cliniquement cette tumeur se présente généralement comme une tuméfaction indolore, de croissance lente et insidieuse. On n'observe ni signes d'atteinte nerveuse, ni adénopathies [10]. A titre anecdotique, un cas de dysphagie due à un myoépithéliome géant du palais mou a été rapporté [4]. En préopératoire des examens tomodensitométriques, échographiques [11] et sialographiques peuvent déterminer avec précision l'extension du processus tumoral, dans le cas des glandes accessoires à la muqueuse et aux tissus profonds, sachant que l'atteinte osseuse est exceptionnelle [12]. Dans le cas présent, un examen tomodensitométrique du palais osseux a été proposé mais, par manque de motivation, le patient l'a refusé. 
Lors de sa découverte, sa taille mesure de 1 à $5 \mathrm{~cm}$ [13]. De consistance ferme sans être dure, le myoépithéliome bénin est bien circonscrit. Sa surface est discrètement lobulée. En siège buccal, l'ulcération est rare sauf en cas d'interférence prothétique ou de traumatismes répétés [4]. Sur la tranche de section apparaît d'ordinaire une capsule parfois interrompue [14] et qui peut manquer.

Tout l'intérêt de la notion de myoépithéliome réside dans les difficultés que recèle son diagnostic anatomopathologique. Deux questions se posent, dont la réponse est loin d'être aisée. L'une est académique : s'agit-il d'un myoépithéliome? L'autre est pratique, et le suivi thérapeutique en dépend : s'agit-il d'une tumeur bénigne, distincte du carcinome myoépithélial ou de toute autre variété de cancer salivaire avec cellules myoépithéliales ? Avant de discuter de ces deux points, les conditions du diagnostic doivent être rappelées. Comme c'est habituellement le cas pour les tumeurs des glandes salivaires, c'est sur la pièce d'exérèse que le diagnostic est porté (et l'a été chez notre patient) : la biopsie n'est pas contributive dans les myoépithéliomes en raison de la variabilité de leur structure d'un territoire à l'autre, d'une lésion à l'autre, et de l'existence d'une population myoépithéliale parfois fort abondante [6, 10]. Cet aspect peut être également observé dans des tumeurs salivaires de pronostic différent de celui du myoépithéliome, d'où l'impossibilité d'établir un diagnostic précis car on a souvent un aspect trompeur ou insuffisant sur le matériel biopsique. A notre sens, la biopsie extemporanée est à proscrire pour les mêmes raisons, tandis que l'examen extemporané de recoupes chirurgicales est au contraire d'un intérêt crucial en pathologie salivaire.

Le myoépithéliome se définit et s'identifie morphologiquement comme une prolifération homogène de cellules myoépithéliales qui sont des constituants normaux de l'assise périphérique des canaux intercalaires et à moindre degré des acini salivaires. Dans le myoépithéliome, les cellules myoépithéliales sont entourées par un stroma œdémateux ou fibro-hyalin [10] et leur apparence varie, pouvant être plas- mocytoïde (ce qui était majoritairement le cas chez notre patient), fusiforme, épithélioïde ou claire $[3,13,15]$. Dans les tumeurs des glandes accessoires, les cellules sont plutôt plasmocytoïdes, dans celles de la parotide, elles sont plutôt fusiformes; ces considérations relatives au type cytologique paraissent n'avoir aucune incidence pronostique [4]. Ceci étant, la nature myoépithéliale de ces différentes inflexions, suggérée par la morphologie, est affirmée par l'immunohistologie $[1-3,10]$ où, à coté des marquages de la PS-100, la vimentine et la GFAP (glial fibrillary acid protein) la coexistence du marquage pour la kératine et le muscle lisse est bien entendu le plus convaincant sur le plan physiopathologique et histopathogénétique. A côté des cellules myoépithéliales, certains auteurs considèrent que l'on peut retrouver des cellules épithéliales formant des structures canalaires mais qui ne dépassent pas $10 \%$ de l'ensemble la lésion $[2,10,16]$. De telles structures s'observaient focalement dans la tumeur actuelle.

Le diagnostic différentiel implique les très nombreuses lésions salivaires renfermant des cellules myoépithéliales. Parmi les lésions bénignes, la plus proche du myoépithéliome est l'adénome pléomorphe. De fait, les myoépithéliomes ont été longtemps classés par les anatomo-pathologistes parmi ces tumeurs [3, 14], dont ils ont le risque de récidive, avec cependant une probabilité plus faible [1]. La présence d'un stroma chondroïde ou myxoïde, même minime et focal, identifie l'adénome pléomorphe. Outre la forme bénigne de myoépithéliome décrite ci-dessus, la forme maligne ou carcinome myoépithélial a également été identifié. Cette forme maligne peut survenir «de novo», apparaître secondairement sur un myoépithéliome bénin (cas le plus fréquent) ou sur un adénome pléomorphe [10]. Relativement rare, le carcinome myoépithélial représente moins de $1 \%$ des tumeurs malignes des glandes salivaires $[17,18]$. L'atteinte est généralement plus tardive que la forme bénigne (62 ans en moyenne), sans prédilection sexuelle [10]. Surtout parotidien, il se présente pourtant une fois sur quatre en siège muqueux et surtout palatin. II a les caractères d'une tumeur médecine buccale chirurgie buccale VOL. $9, \mathrm{~N}^{\circ}$ 2003 page 117 
maligne: absence de capsule, zones de nécrose [3, 13]. L'identification histologique est parfois fort difficile (atypies nucléaires parfois très discrètes, présence de mitoses éventuellement rares). L'élément clef du diagnostic est l'envahissement du tissu salivaire voisin [3]. L'identification des tumeurs malignes avec composante de cellules myoépithéliales se fait par la présence de structures associées, prolifération épithéliale dans le carcinome épithélialmyoépithélial, structures cribriformes dans le

\section{RÉFÉRENCES}

1-Seifert G, Sobin LH. The World Health Organization's Histological Classification of Salivary Gland Tumors. A commentary on the second edition. Cancer 1992; 70: 379-85.

médecine buccale chirurgie buccale

2 - Simpson RH, J ONES H, BeAsLey P. Benign myoepithelioma of the salivary glands: a true entity? Histopathology 1995; 27: 1-9.

3 - ELLIS GL, AUCLAIR P. Tumors of the salivary glands. Atlas of tumor pathology, 3rd series, fascicle 17, Armed Forces Institute of Pathology, Washington DC, 1996.

4 - TAYLOR J, TIGHE J V. A minor salivary gland tumour carcinome adénoïde kystique, pour ne citer que les plus communes [1, 3]. Enfin, le diagnostic différentiel de ces tumeurs doit également inclure les tumeurs contenant des cellules musculaires lisses (leiomyomes), celles d'origine nerveuse et périnerveuse, et enfin les plasmocytomes $[16,19]$. L'intervention chirurgicale consiste en l'exérèse large de la tumeur évitant le risque de récidive et de transformation maligne [20]. Enfin, une surveillance régulière est particulièrement indiquée. presenting with dysphagia. J Laryngol Otol 1999; 113: $569-72$.

5 - SCIUBba J J, Brannon RB. Myoepithelioma of salivary glands : report of 23 cases. Cancer 1982; 49: 562 72.

6 - BigotTI G, Di Giorgio GG. Myoepithelioma of the breast : histologic, immunologic and electromicroscopic appearance. Ultrastruct Pathol 1995; 19: 269-74.

7 - Kilpatrick SE, Hitchcock MG, Kraus MD, Calonje E, FLETCHER CD. Mixed tumors and myoepitheliomas of soft tissue : a clinicopathologic study of 19 cases with a unifying concept. Am J Surg Pathol 1997; 21: 13-22.

8 - Kutzner H, Mentzel T, Kaddu S, Soares LM, Sangueza OP, Requena L. Cutaneous myoepithelioma, an under-recognized cutaneous neoplasm composed of myoepithelial cells. Am J Surg Pathol 2001; 25: 348-55.

9 - Martinez-Madrigal F, Santiago Payan H, Meneses A, Dominguez Malagon H, Rojas ME. Plasmocytoïd myoepithelioma of the laryngeal region: a case report. Hum Pathol 1995; 26: 802-4.

10 - Alos L, Cardesa A, B ombi J A, Mallofre C, Cuchi A, TRASERRA J . Myoepithelial tumors of salivary glands: a clinicopathologic, immunohistochemical, ultrastructural, and flow-cytometric study. Semin Diagn Pathol 1996; 13: 138-47.

11 - Ishil J, Nagasawa $H$, Wadamori T, Yamashiro M, IshiKaWA $H$, Yamada T, MiYakuRa T, Amagasa $T$. Ultrasonography in the diagnosis of palatal tumors. Oral Surg Oral Med Oral Pathol Oral Radiol Endod 1999; 87: 39-43.

12 - Hiwatashi A, Matsumoto S, Kamol I, Yamashita $H$, NAKASHIMA A.Imaging features of myoepithelioma arising from the hard palate. A case report. Acta Radiol 2000; 41: 417-9.

13 - Skalova A, Michal M, Ryska A, Simpson RHW, Kinkor Z, W ALTER J, LeIVO I. Oncocytic myoepithelioma and pleomorphic adenoma of salivary glands. Virchows Arch 1999; 434: 537-46.

14-Barnes L, Appel BN, Perez H, El-Attar am. Myoepithelioma of the head and neck : case report and review. J Surg Oncol 1985; 28: 21-8.

15 - Suglura R, Kuyama K, Utsunomiya T, Motikawa M, Fukumoto M, Yамамото $H$. Myoepithelioma arising from the buccal gland : histopathological and immunohistochemical studies. J Oral Sci 2000; 42: 39-42.

16 - Dardick I. Myoepithelioma : definitions and diagnostic criteria. Ultrastruct Pathol 1995; 19: 335-45.

17 - Auclair PL, ElLIS GL. Atypical features in salivary gland mixed tumors : their relationship to malignant transformation. Mod Pathol 1996; 9: 652-7.

18 - Savera AT, Sloman A, Huvos AG, Klimstra DS. Myoépithélial carcinoma of the salivary glands : a clinicopathologic study of 25 patients. Am J Surg Pathol 2000; 24: 761-74.

19 - De las Casas le, Hoerl hD, Oberley TD, Hafez GR, Sempf JM, Shalkham JE, Kurtycz DF. Myoepithelioma presenting as a midline cystic tongue lesion : cytology, histology, ancillary studies, and differential diagnosis. Diagn Cytopathol 2001; 24: 403-7.

20 - Pogrel MA. The management of salivary gland tumors of the palate. J Oral Maxillofac Surg 1994; 52: 454-9. 